\title{
Biomechanical function of the anterolateral ligament of the knee: a systematic review
}

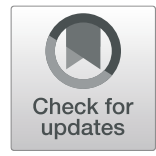

\author{
Jin Kyu Lee ${ }^{1 \dagger}$, Young Jin $\mathrm{Seo}^{2+}$, Soo-Young Jeong ${ }^{1}$ and Jae-Hyuk Yang ${ }^{3 *}$
}

\begin{abstract}
Background: It has been suggested that the anterolateral ligament (ALL) is an important anterolateral stabilizer of the knee joint which functions to prevent anterolateral subluxation and anterior subluxation at certain flexion angles in the knee.

Purpose: To analyze and systematically interpret the biomechanical function of the ALL.

Methods: An online search was conducted for human cadaveric biomechanical studies that tested function of the ALL in resisting anterolateral subluxation and anterior subluxation of the knee. Two reviewers independently searched Medline, Embase, and the Cochrane Database of Systematic Reviews for studies up to 25 September 2018. Biomechanical studies not reporting the magnitude of anterior tibial translation or tibial internal rotation in relation to the function of the ALL were excluded.

Results: Twelve biomechanical studies using human cadavers evaluating parameters including anterior tibial translation and/or internal tibial rotation in anterior cruciate ligament (ACL)-sectioned and ALL-sectioned knees were included in the review. Five studies reported a minor increase or no significant increase in anterior tibial translation and internal tibial rotation with further sectioning of the ALL in ACL-deficient knees. Five studies reported a significant increase in knee laxity in tibial internal rotation or pivot shift with addition of sectioning the ALL in ACL-deficient knees. Two studies reported a significant increase in both anterior tibial translation and internal tibial rotation during application of the anterior-drawer and pivot-shift tests after ALL sectioning.

Conclusion: There was inconsistency in the biomechanical characteristics of the ALL of the knee in resisting anterolateral and anterior subluxation of the tibia.
\end{abstract}

Keywords: biomechanical function, anterolateral ligament, knee, systematic review

\section{Introduction}

The existence of a specific structure in the knee's lateral capsule was discovered by dissections performed in 1879 by Paul Segond. He described it as "a resistant, pearly, fibrous band, which, in an exaggeration of internal rotational movement, is always subjected to an extreme degree of tension" as well as an avulsion fracture now named the "Segond fracture." [1] This "recently" described structure was named the "anterolateral ligament" (ALL) by Vieira et al. [2] in 2007. The ALL has been the subject of many recent publications although there has

\footnotetext{
* Correspondence: jaekorea@gmail.com

${ }^{\dagger}$ Jin Kyu Lee and Young Jin Seo contributed equally to this work.

${ }^{3}$ Department of Orthopedic Surgery, Guri Hospital, Hanyang University

Medical Center, 153, Gyeongchunro, Guri, Gyeonggi-Do 11923, South Korea Full list of author information is available at the end of the article
}

not always been agreement with each other in the anatomic origins $[3,4]$.

Despite recent improvements in surgical methods and understanding of ACL anatomy, it has been suggested that the normal rotational stability of the knee is not fully restored by reconstructive methods for ACL injuries $[5,6]$. Such abnormal biomechanics have led surgeons to focus more on anterolateral structures and, in the past few years, the ALL of the knee has been studied with regard to its anatomy and biomechanics [3, 7-9].

Although several biomechanical studies have been published that the ALL is an important anterolateral stabilizer of the knee joint that prevents anterolateral subluxation (internal tibial rotation) and anterior subluxation at certain flexion angles in the knee [7, 10-12], because of the variability in anatomic descriptions and

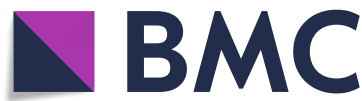

Part of Springer Nature (c) The Author(s). 2020 Open Access This article is distributed under the terms of the Creative Commons Attribution 4.0 International License (http://creativecommons.org/licenses/by/4.0/), which permits unrestricted use, distribution, and reproduction in any medium, provided you give appropriate credit to the original author(s) and the source, provide a link to the Creative Commons license, and indicate if changes were made. The Creative Commons Public Domain Dedication waiver (http://creativecommons.org/publicdomain/zero/1.0/) applies to the data made available in this article, unless otherwise stated. 
methodology in biomechanical testing, some concepts regarding the biomechanical function of the ALL are controversial.

Given the relative paucity of literature reviewing the native biomechanics of the ALL of the knee, the purpose of the present study was to provide a systematic review of the biomechanical ALL function excluding the surgical aspect of ACL and/or ALL. The hypothesis of this study was that there would be inconsistent results in the biomechanical characteristics of the ALL of the knee in resisting anterolateral and anterior subluxation of the tibia.

\section{Methods}

\section{Identification and selection of articles}

Two reviewers independently searched Medline, Embase, and the Cochrane Database of Systematic Reviews for studies up to 25 September 2018. The following search protocol was used: "anterolateral ligament" [All Fields] OR "anterior lateral ligament" [All Fields] OR "ALL" [All Fields] OR "Segond fracture" [All Fields] OR "lateral capsular ligament" [All Fields]) OR "anterior oblique band" [All Fields]) OR "iliotibial tract" [All Fields] AND "biomechanical study" [All Fields]) OR "biomechanical phenomena" [All Fields]) OR "biomechanical phenomena" [All Fields] OR "phenomena, biomechanical" [All Fields] OR "kinematics" [All Fields] OR "biomechanical assessment" [All Fields]) OR "biomechanical function" [All Fields]) OR "biomechanically" [All Fields]) OR "cadaver" [Mesh] OR "cadaver" [All Fields] OR "cadavers" [All Fields] OR "human cadaveric study" [All Fields]) OR "cadaver study" [All Fields].

The inclusion criteria were English language, human cadaveric study, and biomechanics or biomechanical studies on the function of the ALL of the knee. The exclusion criteria were studies on (reconstructive or tenodesis) surgical outcomes; anatomic and radiographic studies of the ALL of the knee; biomechanical studies not reporting the magnitude of anterior tibial translation or tibial internal rotation; review or commentary articles. Duplicated articles were excluded, and two independent reviewers studied the abstracts from all searched articles. After initial identification of articles, full-text review of the selected studies was undertaken.

\section{Data collection}

Study data were collected, specifically those detailing the: torque applied; sequence of the experimental protocol; magnitude of anterior tibial translation and/or internal tibial rotation; other relevant reported results. Quality assessment of the studies was not done because all biomechanical studies did not have associated level of evidence.

\section{Results}

The literature search identified 245 articles. Review of the titles and abstracts excluded 76 duplicates as well as 149 studies not related to biomechanical study of the ALL of the knee. This strategy left 20 articles for fulltext review. Three studies focusing on the wrong topic, two studies related to the outcome of surgical reconstruction, and four studies not reporting the degree of anterior tibial translation and/or internal tibial rotation were excluded, thereby leaving eleven articles. A manual search for reference lists identified one additional article, so twelve articles were finally included for systematic review. (Fig. 1) Risk of bias assessment was done for each included study (Table 1).

Twelve biomechanical studies using human cadavers evaluating parameters including anterior tibial translation and/or internal tibial rotation in ACL-sectioned and ALL-sectioned knees are summarized in Table 2. Five studies (out of the 12 studies reviewed) reported a minor increase or no significant increase in anterior tibial translation and internal tibial rotation with further sectioning of the ALL in ACL-deficient knees $[13,14,16,17,19]$. The remaining 5 studies reported a significant increase in either the anterior translation and knee laxity in tibial internal rotation or pivot shift with addition of ALL sectioning in ACL-deficient knees [7, 10-12, 20]. Two studies reported a significant increase in both anterior tibial translation and internal tibial rotation during application of the anterior-drawer and pivot-shift tests after ALL sectioning $[15,18]$.

\section{Discussion}

This systematic review of the biomechanical studies of the ALL of the knee revealed inconsistencies due to following factors. First, the threshold value for statistical significance was different. If adopting a threshold value of $<3 \mathrm{~mm}$ for anterior tibial translation and $<2^{\circ}$ for internal tibial rotation, as suggested by Noyes et al. [13], some of studies would have been interpreted differently. Second, the magnitude of torque applied and the position of the knees for simulating Lachman and pivot-shift tests were inconsistent. Five of the 12 studies did not demonstrate the significance of ALL both for anterior translation and internal tibia rotation $[13,14,16,17,19]$. While other 5 studies showed either the tibia internal rotation or anterior translation effect after ALL sectioning [7, 10-12, 20], 2 studies showed the statistical significance both in anterior translation and internal tibia rotation $[15,18]$. In addition, management of ITB was different among studies.

Biomechanical studies included in this systematic review had a common experimental protocol with regard to the sequence of ligament sectioning. All biomechanical studies compared the degree of anterior 


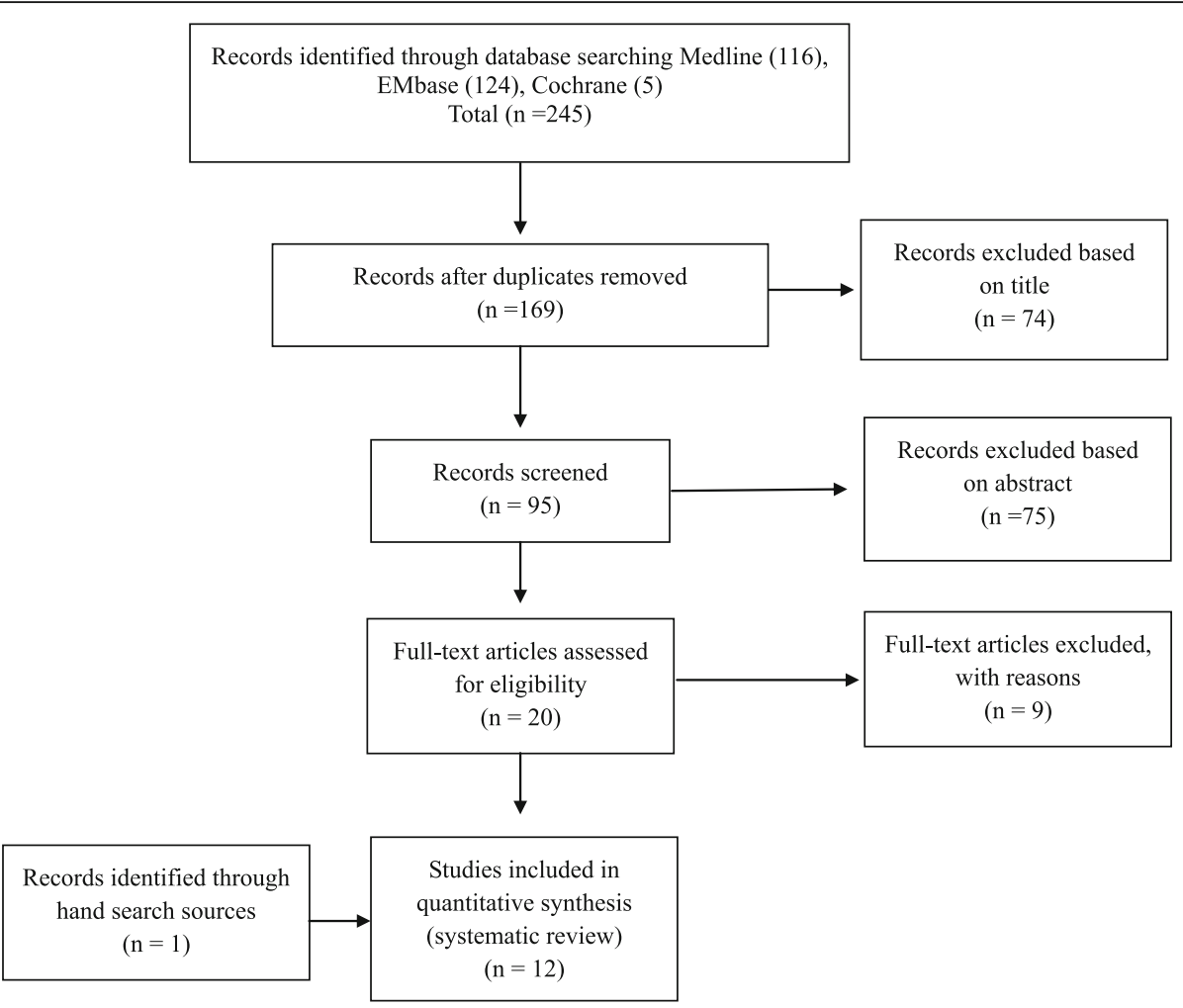

Fig. 1 Preferred reporting items for systematic review flowchart showing application of selection criteria to the studies identified with the search strategy

Table 1 Risk of bias assessment for interrupted time series studies

\begin{tabular}{|c|c|c|c|c|c|c|c|}
\hline & $\begin{array}{l}\text { Intervention } \\
\text { independent of } \\
\text { other changes }\end{array}$ & $\begin{array}{l}\text { Shape of the } \\
\text { intervention effect } \\
\text { pre-specified }\end{array}$ & $\begin{array}{l}\text { Intervention unlikely } \\
\text { to affect the data } \\
\text { collection }\end{array}$ & $\begin{array}{l}\text { Knowledge of allocated } \\
\text { interventions adequately } \\
\text { prevented during the study }\end{array}$ & $\begin{array}{l}\text { Incomplete outcome } \\
\text { data adequately }\end{array}$ & $\begin{array}{l}\text { Selective } \\
\text { outcome } \\
\text { reporting }\end{array}$ & $\begin{array}{l}\text { Other risks } \\
\text { of bias }\end{array}$ \\
\hline $\begin{array}{l}\text { Noyes et al. } \\
2017 \text { [13] }\end{array}$ & L & $\mathrm{L}$ & $\mathrm{L}$ & $\mathrm{H}$ & $U$ & L & $L$ \\
\hline $\begin{array}{l}\text { Inderhaug } \\
\text { et al. } 2017 \text { [10] }\end{array}$ & $\mathrm{L}$ & L & $L$ & $\mathrm{H}$ & $U$ & L & L \\
\hline $\begin{array}{l}\text { Drews et al. } \\
2017 \text { [14] }\end{array}$ & $\mathrm{L}$ & $\mathrm{L}$ & $L$ & $\mathrm{H}$ & U & $\mathrm{L}$ & $U$ \\
\hline $\begin{array}{l}\text { Sonnery-Cottet } \\
\text { et al. } 2016 \text { [15] }\end{array}$ & $\mathrm{L}$ & $\mathrm{L}$ & $\mathrm{L}$ & $\mathrm{H}$ & U & $\mathrm{L}$ & $L$ \\
\hline $\begin{array}{l}\text { Thein et al. } \\
2016 \text { [16] }\end{array}$ & $\mathrm{L}$ & $\mathrm{L}$ & $L$ & $\mathrm{H}$ & U & $\mathrm{L}$ & $L$ \\
\hline $\begin{array}{l}\text { Spencer et al. } \\
2016 \text { [17] }\end{array}$ & $\mathrm{L}$ & $\mathrm{L}$ & $\mathrm{L}$ & $\mathrm{H}$ & U & L & L \\
\hline $\begin{array}{l}\text { Ruiz et al. } \\
2016 \text { [12] }\end{array}$ & L & $\mathrm{L}$ & $L$ & $\mathrm{H}$ & U & $\mathrm{L}$ & L \\
\hline $\begin{array}{l}\text { Rasmussen } \\
\text { et al. } 2016 \text { [18] }\end{array}$ & $\mathrm{L}$ & $L$ & $L$ & $\mathrm{H}$ & U & $\mathrm{L}$ & $L$ \\
\hline $\begin{array}{l}\text { Bonanzinga } \\
\text { et al. } 2016 \text { [7] }\end{array}$ & $\mathrm{L}$ & L & $L$ & $\mathrm{H}$ & U & $\mathrm{L}$ & $U$ \\
\hline $\begin{array}{l}\text { Saiegh et al. } \\
2015 \text { [19] }\end{array}$ & $\mathrm{L}$ & $L$ & $L$ & $\mathrm{H}$ & U & $\mathrm{L}$ & $U$ \\
\hline $\begin{array}{l}\text { Parsons et al. } \\
2015 \text { [20] }\end{array}$ & $\mathrm{L}$ & $\mathrm{L}$ & $\mathrm{L}$ & $\mathrm{H}$ & U & L & $L$ \\
\hline $\begin{array}{l}\text { Monaco et al. } \\
2012[11]\end{array}$ & $\mathrm{L}$ & $\mathrm{L}$ & $\mathrm{L}$ & $\mathrm{H}$ & U & $\mathrm{L}$ & $\mathrm{L}$ \\
\hline
\end{tabular}




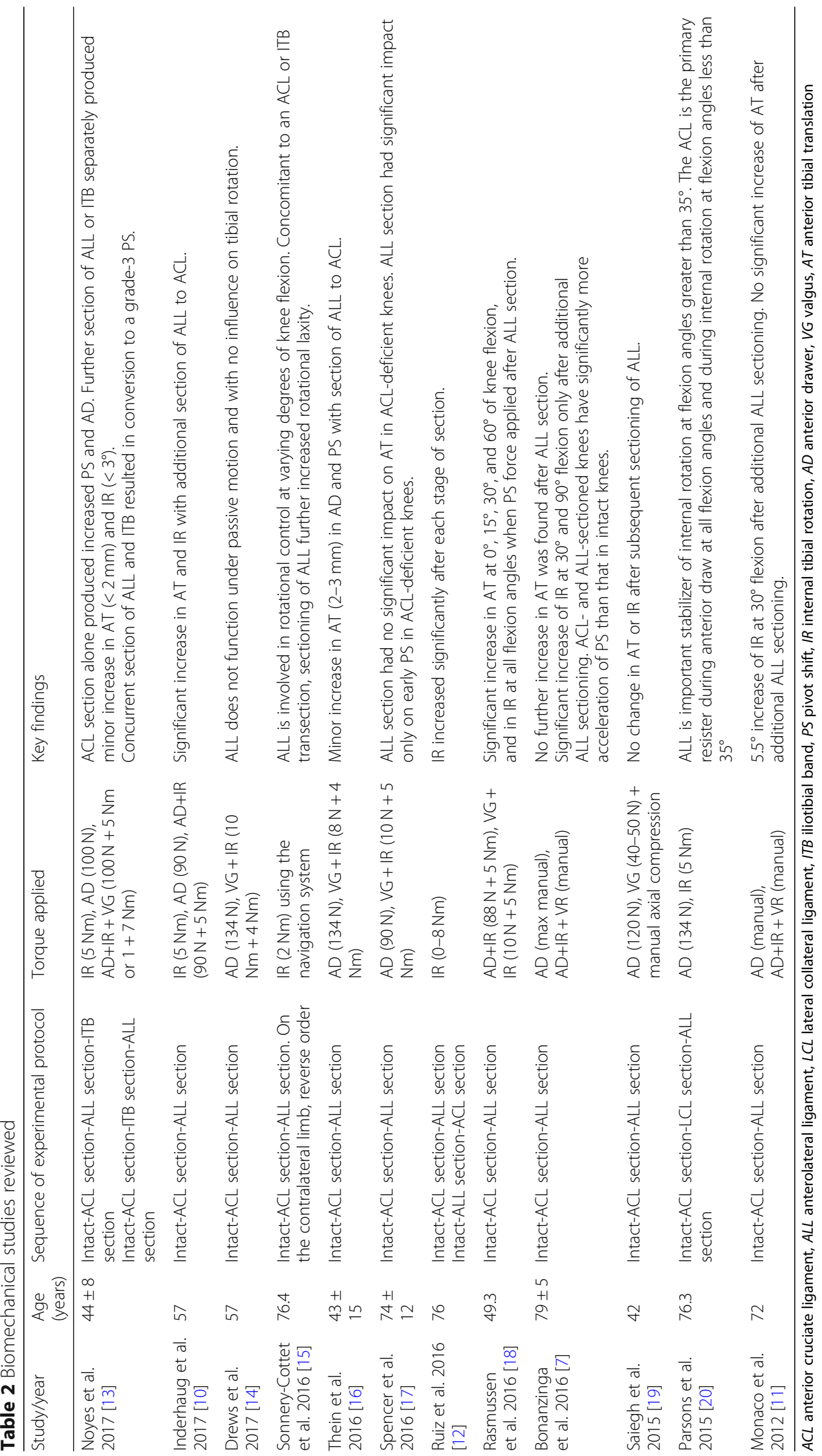


tibial translation and/or internal tibial rotation after sectioning of the ACL followed by sectioning of the ALL [7, 10-20]. The main variation or inconsistencies between studies probably resulted from differences in the torque and maneuvers applied to the cadaveric knees. Universal protocols to simulate the pivot-shift and anterior-drawer tests of the knee are lacking, so such inconsistency may be inevitable.

Five studies (out of the nine reviewed) showed only a minor increase or no significant increase in anterior tibial translation or internal tibial rotation (i.e., pivot shift) with further sectioning of the ALL in ACL-deficient knees. Noyes et al. [13] found only a minor increase in the pivot shift and tibial internal rotations $(<2 \mathrm{~mm}$ or $<$ $3^{\circ}$ ) after ALL or iliotibial band (ITB) sectioning in ACLdeficient knees. They concluded that anatomic ALL or ITB reconstructions would not block a positive pivot shift. However, concurrent sectioning of the ALL and ITB resulted in conversion in most knees to a grade-3 pivot-shift subluxation, suggesting that most biomechanical studies overestimated the function of the ALL by removing ITB. Saiegh et al. [19] also measured anterior tibial translation and internal tibial rotation after sectioning the ALL in ACL-deficient knees. They found increases of only $-0.7 \mathrm{~mm}$ and $0.3^{\circ}$ in the anterior tibial translation and internal tibial rotation, respectively. ITB was also detached in this study. Spencer et al. [17] reported very similar outcomes with two prior studies, reporting an increase of only $2^{\circ}$ in internal tibial rotation after additional ALL sectioning in ACL-deficient knees. ITB was not damaged in their study by incising the posterior border of longitudinal fibers and retracting anteriorly. It has been suggested that $99 \%$ of chronic ACL ruptures and $95 \%$ of acute ACL ruptures display a difference of $\geq 3 \mathrm{~mm}$ in anterior tibial translation, so $2 \mathrm{~mm} \mathrm{(<}$ $3 \mathrm{~mm}$ ) had been chosen as the threshold for a significant difference in previous studies [13, 21, 22]. Thein et al. [16] reported similar results to the previous studies because they found a mean increase of $2-3 \mathrm{~mm}$ (i.e., within the threshold value) in anterior tibial translation in anterior-stability and simulated pivot-shift tests. Furthermore, the load borne by the ALL in ACL-intact knees was minimal in response to simulated pivot-shift and anterior-drawer tests. They concluded that the ALL is a secondary stabilizer to the ACL whereby only the ALL bears increased loads at extremes of tibial translation in ACL-deficient knees. ITB was reflected in this series.

In contrast, five studies reported a significant increase in knee laxity either in tibial internal rotation or pivot shift with addition of ALL sectioning in ACL-deficient knees. Inderhaug et al. [10] found a significant increase in knee laxity by adding an anterolateral lesion to ACLdeficient knees when an anterior drawer force and internal tibial torque was applied. Furthermore, they identified a significant restoration of knee laxity when a combined lateral tenodesis and MacIntosh procedure were combined to ACL reconstruction. However, their study was limited because clinically significant threshold values were not applied. The ITB was transected in this series. Conversely, Monaco et al. [11] reported a significant increase in internal tibial rotation of $5.5^{\circ}$ at a knee flexion of $30^{\circ}$ after ALL sectioning, although there was no significant increase in anterior tibial translation after additional ALL sectioning. ITB was not damaged in their study by incising the longitudinal fibers and retracting anteriorly. Bonanzinga et al. [7] reported a significant increase in tibial internal rotation at $30^{\circ}$ and $90^{\circ}$ of knee flexion after additional sectioning of the ALL. They also found increased acceleration of the pivot shift if the ACL and ALL were sectioned compared with the intact state. The ITB was separated longitudinally. Ruiz et al. [12] applied only an internal rotation force without the anterior-drawer test, and reported a significant additional increase in internal tibial rotation regardless of the sequence of sectioning between the ACL and ALL. The ITB was incised longitudinally in this series.

Two studies reported a significant increase in both anterior tibial translation and internal tibial rotation during anterior-drawer and pivot-shift tests after ALL sectioning $[15,18]$. Robotic biomechanical testing was undertaken, and a significant increase in anterior tibial translation was found at $0^{\circ}, 15^{\circ}, 30^{\circ}$, and $60^{\circ}$ of knee flexion, and in internal tibial rotation at all flexion angles when the pivot shift force was applied [18]. However, similar to other studies, clinically significant threshold values were not applied. Management of ITB was not described in detail. Sonnery-Cottet et al. [15] tested the cadaveric knees in internal rotation at $20^{\circ}$ and $90^{\circ}$ of flexion and then subsequently tested using a simulated pivot-shift test consisting of coupled axial rotation at $30^{\circ}$ of flexion. Serial sectioning of the ACL, ALL, and ITB was performed. After ACL sectioning, an incision of the ALL induced a significant increase in internal rotation $\left(119.2 \%[P=.0002]\right.$ at $20^{\circ} ; 121.8 \%[P=.0029]$ at $\left.90^{\circ}\right)$ and in coupled axial rotation $(143.0 \% ; P=.0035)$ compared with the intact knee as well as a significant increase in internal rotation at $90^{\circ}(113.4 \% ; P=.009)$ and in coupled axial rotation $(130.8 \% ; P=.0124)$ compared with the ACL deficient knee. They concluded that the ALL is involved in rotational control of the knee at varying degrees of knee flexion and during a simulated pivot shift. They also mentioned that the concomitant to an ACL or ITB transection, sectioning the ALL further increased rotational laxity.

This systematic review has a few limitations. First, the heterogeneous protocols and experimental settings of biomechanical studies and different threshold values limited the scope of direct comparisons for biomechanical results. 
Second, several biomechanical studies were excluded because of different assessment parameters and status of knees (i.e., surgically reconstructed). Tavlo et al. [23] undertook a similar biomechanical cadaveric study but, after ACL reconstruction, found no significant difference between an intact and sectioned ALL with regard to anterior tibial translation.

\section{Conclusion}

There were inconsistent results among studies in the biomechanical characteristics of the ALL of the knee in resisting anterolateral and anterior subluxation of the tibia.

\section{Acknowledgements}

Jin Kyu Lee and Young Jin Seo contributed equally.

\section{Authors' contributions}

Conceived ideas or experimental design: JKL, YJS, JHY. Performed experiments/data collection: JKL, YJS, JHY. Data analysis and interpretation: $J K L, S Y J$, and JHY. Primary author: JKL, JHY SYJ, and JHY.

\section{Funding}

None.

\section{Availability of data and materials \\ Possible.}

\section{Competing interests}

No benefits in any form have been received or will be received from a commercial party related directly or indirectly to the subject of this article.

\section{Author details}

'Department of Orthopaedic Surgery, Hanyang University Hospital, Seoul, South Korea. ${ }^{2}$ Department of Orthopaedic Surgery, Dongtan Sacred Heart Hospital, Dongtan, Gyeonggi-do, South Korea. ${ }^{3}$ Department of Orthopedic Surgery, Guri Hospital, Hanyang University Medical Center, 153,

Gyeongchunro, Guri, Gyeonggi-Do 11923, South Korea.

Received: 14 June 2019 Accepted: 21 November 2019

Published online: 01 January 2020

\section{References}

1. Segond P-F (1879) Recherches cliniques et expérimentales sur les épanchements sanguins du genou par entorse. Prog Med 7:297-299 319-21, 40-41

2. Vieira EL, Vieira EA, da Silva RT, Berlfein PA, Abdalla RJ, Cohen M (2007) An anatomic study of the iliotibial tract. Arthroscopy. 23:269-274

3. Claes S, Vereecke E, Maes M, Victor J, Verdonk P, Bellemans J (2013) Anatomy of the anterolateral ligament of the knee. J Anat 223:321-328

4. Helito CP, Bonadio MB, Rozas JS, Wey JM, Pereira CA, Cardoso TP, Pecora JR, Camanho GL, Demange MK (2016) Biomechanical study of strength and stiffness of the knee anterolateral ligament. BMC Musculoskelet Disord 17:193

5. Di Benedetto P, Di Benedetto E, Fiocchi A, Beltrame A, Causero A (2016) Causes of Failure of Anterior Cruciate Ligament Reconstruction and Revision Surgical Strategies. Knee Surg Relat Res 28:319-324

6. Wroble RR, Grood ES, Cummings JS, Henderson JM, Noyes FR (1993) The role of the lateral extraarticular restraints in the anterior cruciate ligamentdeficient knee. Am J Sports Med 21:257-262 discussion 63

7. Bonanzinga T, Signorelli C, Grassi A, Lopomo N, Bragonzoni L, Zaffagnini S, Marcacci M (2017) Kinematics of ACL and anterolateral ligament. Part I: Combined lesion. Knee Surg Sports Traumatol Arthrosc 25:1055-1061

8. Chambat P, Guier C, Sonnery-Cottet B, Fayard JM, Thaunat M (2013) The evolution of $A C L$ reconstruction over the last fifty years. Int Orthop 37:181-186

9. Syam K, Chouhan DK, Dhillon MS (2017) Outcome of ACL reconstruction for chronic ACL injury in knees without the posterior horn of the medial meniscus: comparison with $A C L$ reconstructed knees with an intact medial meniscus. Knee Surg Relat Res 29:39-44

10. Inderhaug E, Stephen JM, Williams A, Amis AA (2017) Biomechanical comparison of anterolateral procedures combined with anterior cruciate ligament reconstruction. Am J Sports Med 45:347-354

11. Monaco E, Ferretti A, Labianca L, Maestri B, Speranza A, Kelly MJ, D'Arrigo C (2012) Navigated knee kinematics after cutting of the ACL and its secondary restraint. Knee Surg Sports Traumatol ArthrosC 20:870-877

12. Ruiz N, Filippi GJ, Gagniere B, Bowen M, Robert HE (2016) The comparative role of the anterior cruciate ligament and anterolateral structures in controlling passive internal rotation of the knee: a biomechanical study. Arthroscopy. 32:1053-1062

13. Noyes FR, Huser LE, Levy MS (2017) Rotational knee instability in ACLdeficient knees: role of the anterolateral ligament and iliotibial band as defined by tibiofemoral compartment translations and rotations. J Bone Joint Surg Am 99:305-314

14. Drews BH, Kessler O, Franz W, Durselen L, Freutel M (2017) Function and strain of the anterolateral ligament part I: biomechanical analysis. Knee Surg Sports Traumatol Arthrosc 25:1132-1139

15. Sonnery-Cottet B, Lutz C, Daggett M, Dalmay F, Freychet B, Niglis L, Imbert $P$ (2016) The involvement of the anterolateral ligament in rotational control of the knee. Am J Sports Med 44:1209-1214

16. Thein R, Boorman-Padgett J, Stone K, Wickiewicz TL, Imhauser CW, Pearle AD (2016) Biomechanical assessment of the anterolateral ligament of the knee: a secondary restraint in simulated tests of the pivot shift and of anterior stability. J Bone Joint Surg Am 98:937-943

17. Spencer L, Burkhart TA, Tran MN, Rezansoff AJ, Deo S, Caterine S, Getgood AM (2015) Biomechanical analysis of simulated clinical testing and reconstruction of the anterolateral ligament of the knee. Am J Sports Med 43:2189-2197

18. Rasmussen MT, Nitri M, Williams BT, Moulton SG, Cruz RS, Dornan GJ, Goldsmith MT, LaPrade RF (2016) An in vitro robotic assessment of the anterolateral ligament, part 1: secondary role of the anterolateral ligament in the setting of an anterior cruciate ligament injury. Am J Sports Med 44: 585-592

19. Saiegh YA, Suero EM, Guenther D, Hawi N, Decker S, Krettek C, Citak M, Omar M (2017) Sectioning the anterolateral ligament did not increase tibiofemoral translation or rotation in an ACL-deficient cadaveric model. Knee Surg Sports Traumatol Arthrosc 25:1086-1092

20. Parsons EM, Gee AO, Spiekerman C, Cavanagh PR (2015) The biomechanical function of the anterolateral ligament of the knee. Am J Sports Med 43:669-674

21. Gardner EJ, Noyes FR, Jetter AW, Grood ES, Harms SP, Levy MS (2015) Effect of anteromedial and posterolateral anterior cruciate ligament bundles on resisting medial and lateral tibiofemoral compartment subluxations. Arthroscopy. 31:901-910

22. Rangger C, Daniel DM, Stone ML, Kaufman K (1993) Diagnosis of an ACL disruption with KT-1000 arthrometer measurements. Knee Surg Sports Traumatol Arthrosc 1:60-66

23. Tavlo M, Eljaja S, Jensen JT, Siersma VD, Krogsgaard MR (2016) The role of the anterolateral ligament in $\mathrm{ACL}$ insufficient and reconstructed knees on rotatory stability: a biomechanical study on human cadavers. Scand J Med Sci Sports 26:960-966

\section{Publisher's Note}

Springer Nature remains neutral with regard to jurisdictional claims in published maps and institutional affiliations.

Ready to submit your research? Choose BMC and benefit from:

- fast, convenient online submission

- thorough peer review by experienced researchers in your field

- rapid publication on acceptance

- support for research data, including large and complex data types

- gold Open Access which fosters wider collaboration and increased citations

- maximum visibility for your research: over $100 \mathrm{M}$ website views per year

At BMC, research is always in progress.

Learn more biomedcentral.com/submissions 